\title{
Trapped non-equilibrium states in aqueous solutions of oppositely charged polyelectrolytes and surfactants: effects of mixing protocol and salt concentration
}

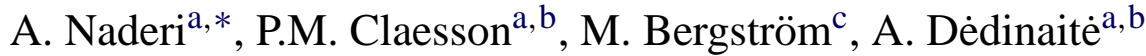 \\ ${ }^{a}$ Department of Chemistry, Surface Chemistry, Royal Institute of Technology, Drottning Kristinas väg 51, SE-100 44 Stockholm, Sweden \\ ${ }^{\mathrm{b}}$ Institute for Surface Chemistry, P.O. Box 5607, SE-114 86 Stockholm, Sweden \\ ${ }^{\mathrm{c}}$ Department of Pharmacy, Box 580, SE-751 23 Uppsala, Sweden
}

Received 9 March 2004; accepted 28 October 2004

Available online 15 December 2004

\begin{abstract}
The very slow equilibration time in oppositely charged systems makes it necessary to control not only the concentration of the species but also the details of the mixing process. This has been demonstrated for processes occurring at interfaces where order of addition effects can be of great importance. In this investigation we set out to study the bulk properties of aqueous mixtures of a highly charged cationic polyelectrolyte mixed with an anionic surfactant with the aim to learn if long-lived non-equilibrium states were formed also in this case, and thus if the details of the mixing procedure would affect the structure of the aggregates formed. For simplicity we chose two mixing protocols, denoted "PTS" and "STP". In the PTS-method the polyelectrolyte is added to the surfactant solution whereas in the STP-method the surfactant is added into the polyelectrolyte solution. The properties of the mixtures in aqueous solutions, with different $\mathrm{NaCl}$ concentrations and as a function of time, were followed by conducting turbidity, electrophoretic mobility and dynamic light scattering measurements. The results demonstrate that the mixing protocol indeed has a great impact on the size of the aggregates initially formed and that this size difference persists for long times. Hence, trapped non-equilibrium states do play an important role also in the bulk solution. We found that in excess surfactant solutions the smaller aggregates formed by the STP-method are more resistant than the larger ones formed by the PTS-method to colloidal instability induced by electrolytes $(\mathrm{NaCl})$. Based on our results we suggest that for producing small and stable polyelectrolyte-surfactant aggregates in systems with excess surfactant, the surfactant should be added last, while the opposite should be applied for systems with excess polyelectrolyte.
\end{abstract}

(C) 2004 Elsevier B.V. All rights reserved.

Keywords: Polyelectrolyte; Surfactant; Polyelectrolyte-surfactant complex; Polyelectrolyte-surfactant aggregate; Polyelectrolyte-surfactant association; Turbidity; Electrophoretic mobility; Dynamic light scattering; Colloidal stability; Non-equilibrium state; Trapped state; Polydispersity

\section{Introduction}

Polymers and surfactants are used together in many applications such as cleaning, emulsification and rheology control to mention but a few, which have resulted in significant technical and scientific interest. A subclass is polyelectrolytes and oppositely charged surfactants, which associate strongly due

\footnotetext{
* Corresponding author. Tel.: +46 8790 9912; fax: +46 8208998 .

E-mail address: ali.naderi@surfchem.kth.se (A. Naderi).
}

to mainly electrostatic and hydrophobic interactions. In some cases the complete phase diagram has been determined [1], but most studies focus on relatively dilute aqueous solutions. The association pattern in this regime can be influenced by moderating the electrostatic attraction by varying salt concentration [2], polyelectrolyte charge density $[3,4]$, or by using mixtures of charged and uncharged surfactants [5]. It can also be moderated by controlling the hydrophobic interaction, for instance by varying the chain length of the surfactant $[6,7]$ and by introducing non-polar sites in the polyelectrolyte structure 
[8]. The architecture of the polyelectrolyte, e.g. the intrinsic stiffness [9] and branching [10,11], influences the way the polyelectrolyte can change conformation in order to promote surfactant self-assembly and is thus also of importance.

As a rule, the association between polyelectrolytes and oppositely charged surfactants starts at very low surfactant concentrations (typically 1-3 orders of magnitude below the critical micelle concentration, CMC, of the surfactant). We call the entity formed by association between one polyelectrolyte and surfactants a polyelectrolyte-surfactant complex, whereas the term "aggregate" is used for association structures containing any number of (also one) polyelectrolyte chains. When the charge of the polyelectrolyte-surfactant aggregate is sufficiently reduced, aggregation will occur readily. At this stage the solution becomes noticeably turbid to the naked eye. However, at still higher surfactant concentrations a clear solution is often, but not always, formed again. A higher charge density of the polyelectrolyte counteracts the formation of a clear solution in presence of excess surfactant, see for instance [12].

It often takes a very long time to reach true equilibrium in strongly associating polymeric systems. For instance when considering polyelectrolytes adsorbed to oppositely charged surfaces, or any polymer showing a high-affinity adsorption isotherm to a surface, it is found that the desorption kinetics are extremely slow. This has lead to the notion of "irreversible adsorption" even though a more correct term in many cases would be an extremely slowly reversible adsorption [13]. The high affinity between polyelectrolytes and oppositely charged surfaces results in a need for control of the experimental pathway in order to obtain reproducible results since the system often becomes trapped in long-lived non-equilibrium states. For instance, it has been shown that the order of addition of inorganic ions and polyelectrolytes affect the structure of adsorbed polyelectrolyte layers [14-16], that is the structure does not only depend on the bulk composition but also on whether the polyelectrolyte or the salt was added first. Similarly Chen et al. [17] showed that the order of addition of two oppositely charged polyelectrolyte solutions determines the end net charge of the system, and that deviations from 1:1 stoichiometry in the formed aggregates increases with ionic strength of the system. This, of course, has important consequences in technical applications.

The state of composite surface layers formed by polyelectrolytes and oppositely charged surfactants are also found to depend on the experimental pathway [18]. In particular Tilton and co-workers have shown how surfactants can be employed to anchor polymers to solid surfaces in long-lived trapped non-equilibrium states [19-24]. Thus, it is clear that trapped non-equilibrium states in complex layers formed on surfaces are prevalent, and understanding these states and how they can be modified by the proper choice of the experimental pathway remains a challenge, even though the understanding has increased during the past years [25].

The common observation of trapped states formed on surfaces naturally leads to the question of whether such states are formed also in complex solutions of oppositely charged species, e.g. oppositely charged polymers or polyelectrolyte-surfactant mixtures. What is known is that in certain concentration ranges very stable and surprisingly monodisperse polyelectrolyte-surfactant aggregates are formed [26,27], and that the internal structures of some of these aggregates are well defined [18,28-32], e.g. consisting of rod-like surfactant micelles wrapped by polyelectrolyte, with the micelles ordered in hexagonal symmetry.

In this work we have investigated the formation of trapped non-equilibrium states in bulk solutions containing a highly charged cationic polyelectrolyte mixed with an anionic surfactant for prolonged times, up to $1000 \mathrm{~h}$. The methods of choice for our investigations were turbidity and electrophoretic mobility measurements combined with dynamic light scattering studies. The polyelectrolyte concentration has been kept constant at $20 \mathrm{ppm}$, whereas the surfactant concentration has been varied up to its CMC-value in pure water $(8.3 \mathrm{mM})$. The salt concentration was also varied to moderate the electrostatic driving force for association and the repulsion between polyelectrolyte-surfactant aggregates. We will compare the results obtained with those for similar mixtures consisting of poly(vinyl amine), a linear cationic polyelectrolyte with ionizable groups, and an anionic surfactant [33].

\section{Materials and methods}

\subsection{Materials}

The polyelectrolyte used was poly\{[2-(propionyloxy) ethyl]trimethylammonium chloride $\}$ (PCMA), which was obtained as a gift from Laboratoire de Physico-Chimie Macromoléculaire, Université Pierre et Marie Curie, Paris. The PCMA sample has a number average molecular weight of $1600 \mathrm{~kg} / \mathrm{mol}$ or about 10,000 segments per molecule. Each segment of the chain carries one permanent positive charge (quaternary ammonium group) located $0.6-0.7 \mathrm{~nm}$ away from the linear backbone. The anionic surfactant sodium dodecyl sulfate (SDS), with $>99 \%$ purity, was obtained from BDH. It was recrystallized two times from ethanol before being used. The sodium chloride $(\mathrm{NaCl})$, with $>99.5 \%$ purity, was obtained from Merck and used as received. The water was first pre-treated with a Milli-RO 10 Plus system and further purified with a Milli-Q PLUS 185 system.

\subsection{Mixing procedures}

To prepare the samples $(30 \mathrm{ml})$ containing $20 \mathrm{ppm}$ PCMA (ca. $0.13 \mathrm{mM}$ charged segments) and different concentrations of SDS, stock solutions of PCMA (1000 ppm) and SDS (8.3 and $16.4 \mathrm{mM}$ ) in solutions with different ionic strength were first made. The samples were prepared by first creating a premix of the aqueous salt solution and either surfactant or polymer, which was blended by turning the sample tube upside down a few times. The last ingredient was then added 
and a final blending was carried out in the same way as described above. Two different mixing methods were employed for preparation of blends of PCMA and SDS, in solutions with different concentration of $\mathrm{NaCl}(0-100 \mathrm{mM})$. The first procedure involved the mixing of SDS and the aqueous salt solution (which constituted the premix) prior to the addition of a small amount of the polyelectrolyte stock solution. This was done slowly in a drop-wise fashion taking ca. $5 \mathrm{~s}$. This procedure will be referred to as PTS (Polyelectrolyte added To the Surfactant). In the second procedure the polyelectrolyte and the aqueous salt solution constituted the premix, to which an amount of the concentrated surfactant solution was then added drop-wise. This process will be referred to as STP (Surfactant added To the Polyelectrolyte).

\subsection{Methods of investigation}

\subsubsection{Turbidity measurements}

The turbidity of PCMA-SDS mixtures containing $20 \mathrm{ppm}$ PCMA, different $\mathrm{NaCl}$ concentrations and various amounts of SDS was measured employing a $\mathrm{HACH}$ ratio turbidimeter. The turbidity of each sample was measured before, $T_{1}$, and after addition of PCMA or SDS, $T_{2}$, depending on the mixing method. The $T_{2}$-values were registered starting $15 \mathrm{~min}$ after the addition of the final ingredient. In this paper we will present the turbidity results as the turbidity difference $\left(\Delta T=T_{2}-T_{1}\right)$ between the PCMA-SDS mixture and the corresponding polyelectrolyte/SDS free solution as a function of the total (bound and free) SDS concentration. We noted that the turbidity prior to mixing the components was always small and that $\Delta T$ is very close to $T_{2}$. Hence in the text the term "turbidity" will be used instead of the more correct but less convenient term "turbidity difference".

\subsubsection{Electrophoretic mobility measurements}

The electrophoretic mobility of the PCMA-SDS aggregates was measured, $15 \mathrm{~min}$ after the preparation of the samples, using a Zetasizer 2000, Malvern Instruments, United Kingdom, employing the standard electrophoresis cell ZET5104. The instrument was calibrated using Malvern Zeta Potential transfer standard (code DTS-1050) prior to use.

\subsubsection{Dynamic light scattering measurements}

Dynamic light scattering measurements were carried out using a BI-200SM goniometer system connected to a BI9000AT digital correlator from Brookhaven Instruments and a water-cooled Lexel 95-2 laser with maximum power of $2 \mathrm{~W}$. The scattered light was measured at an angle of $90^{\circ}$ relative to the incident beam $(\lambda=514 \mathrm{~nm})$. The samples were investigated at room temperature $\left(20-23^{\circ} \mathrm{C}\right)$, and the measurements were carried out no sooner than $15 \mathrm{~min}$ after addition of the last component. For the theory behind light scattering measurements we refer the interested reader to the extensive literature available (e.g. [34]). The Contin method was employed to perform the inverse Laplace transformation analysis.

\section{Results and discussion}

In this section we will first report and discuss the properties of the systems shortly after mixing, and then demonstrate how they evolve with time.

\subsection{Turbidity shortly after mixing}

The turbidity of solutions containing mixtures of PCMA and SDS in solutions with different ionic strengths, prepared according to the different blending methods, and obtained 15 min after their preparation is illustrated in Fig. 1a-d. Before commenting on the results we have to stress that each point, in the curves presented in this paper, is an entity that has been mixed and separately prepared, and therefore is not the result of the continuous increase of the surfactant concentration in one particular sample.

We start by using the data obtained with the STP-method in a salt-free solution, Fig. 1a, to describe the general feature of the curves. It is clear that the turbidity is low at low surfactant concentrations, reaches a maximum at around $0.3 \mathrm{mM}$ SDS, and then decreases again at higher surfactant concentrations. Dedinaite et al. [35] explained the above trend as being due to variations of the net charge of the PCMA-SDS aggregates. We use Fig. 2a to illustrate the reasoning. At SDS concentrations below the turbidity maximum, the aggregates carry a net positive charge (i.e. excess of polyelectrolyte charges), manifested by their positive electrophoretic mobility. Due to electrostatic repulsion, the aggregates are prevented from coming close to each other and form larger aggregates - the turbidity is hence low. At the turbidity maximum (which occurs around $0.3 \mathrm{mM}$ SDS) the electrophoretic mobility reaches zero showing that the PCMA-SDS aggregates are uncharged, thus there are no electrostatic forces present to prevent formation of large aggregates. Above the turbidity maximum the net charge of the aggregates is negative, due to the incorporation of excess SDS. The net negative charge, when generating a sufficiently strong repulsive double-layer force, prevents association between polyelectrolyte-surfactant aggregates in the same manner as described above. We suggest that the excess SDS is incorporated due to hydrophobic interactions between surfactant tails, i.e. the same driving force as for formation of free SDS micelles and for complexes formed between SDS and uncharged polymers. The same reasoning holds for both mixing protocols.

Coming back to Fig. 1, we notice that the way the solutions are prepared has an effect on their turbidity. This effect is most pronounced at the highest surfactant concentrations, where the PTS-method results in significantly higher turbidity than the STP-method. On the other hand, at low surfactant concentrations the STP-method gives higher turbidity as compared to the PTS-method, but in this surfactant concentration range the effect is smaller but it is repeatedly observed at all ionic strengths. We propose that any effect of the mixing procedure, and thus the differences be- 

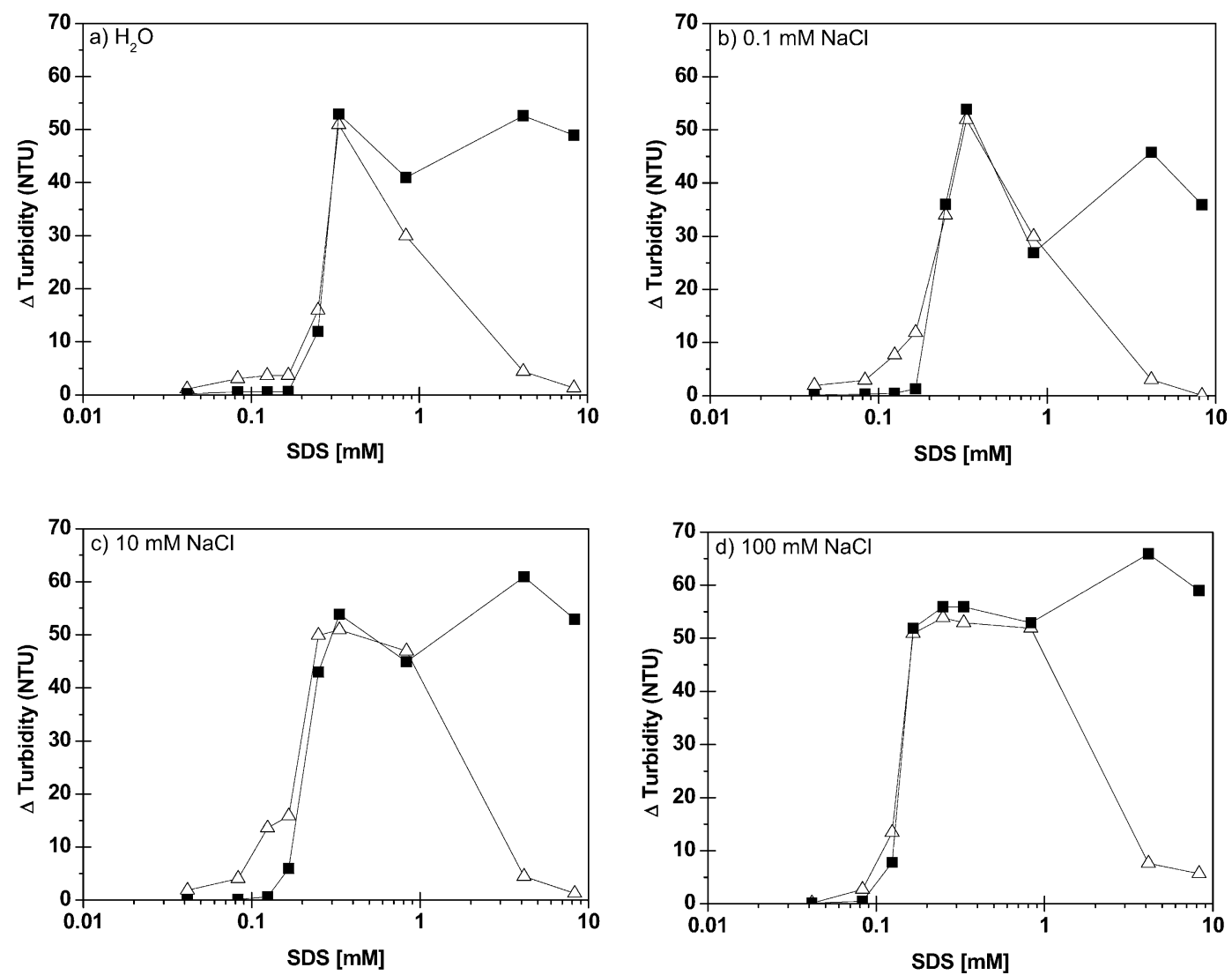

Fig. 1. Turbidity of solutions containing PCMA and SDS measured 15 min after mixing. The solutions were prepared by the STP-method $(\triangle)$ and the PTSmethod (ם). The conditions were no added salt (a, top left), $0.1 \mathrm{mM} \mathrm{NaCl}$ (b, top right), $10 \mathrm{mM} \mathrm{NaCl}$ (c, bottom left) and $100 \mathrm{mM} \mathrm{NaCl}$ (d, bottom right). The PCMA concentration was $20 \mathrm{ppm}$.

tween the PTS and STP-methods, is due to events occurring before the surfactant and polymer have become distributed homogeneously in the whole solution volume. Thus, when one adds a concentrated surfactant solution to a homogeneous polyelectrolyte solution, as in the STP-method, the temporary and locally high surfactant concentration may give rise to non-equilibrium effects. This is not a problem at high surfactant concentrations, when colloidally stable aggregates with excess surfactants are formed. However, the locally high surfactant concentration is likely to cause aggregation close to the instability boarder at low surfactant concentrations since locally some charge-neutral stoichiometric complexes may form and flocculate into larger aggregates. This explains the higher turbidity for the STP-method compared to the PTS-method at low surfactant concentrations. On the other hand, when a concentrated polyelectrolyte solution is added to a homogeneous surfactant solution, the PTS-method, the initial and locally high polymer concentration may give rise to non-equilibrium effects. This is not a problem at low surfactant concentrations where the aggregates formed have positive charge excess. However, at high surfactant concentrations the locally high polymer concentration may result in the formation of large multi- chain aggregates during the association process with the surfactants, i.e. prior to that the aggregates have obtained a net negative charge. This would explain the high turbidity at high surfactant concentrations observed with the PTSmethod.

One more point regarding the data presented in Fig. 1 deserves some comments. First, we recognize that by increasing the salt concentration we achieve a broadening of the concentration range over which both the STP- and PTSmethods result in high turbidity solutions. This is consistent with the results obtained for the PVAm-SDS system [33], except for one feature - the broadening of the high turbidity region achieved by adding salt is higher for the PCMA-SDS system. Apparently, the observation that addition of salt results in increased aggregation (as seen by the higher turbidity) is true in both cases. However, the details are dependent on the structure of the polyelectrolyte and the structure of the complexes and aggregates formed.

Finally we note that the extent to which non-equilibrium states are formed is likely to depend on the time scale over which the components distribute evenly throughout the whole solution compared to the time scales of polyelectrolyte-surfactant complex formation and their sub- 

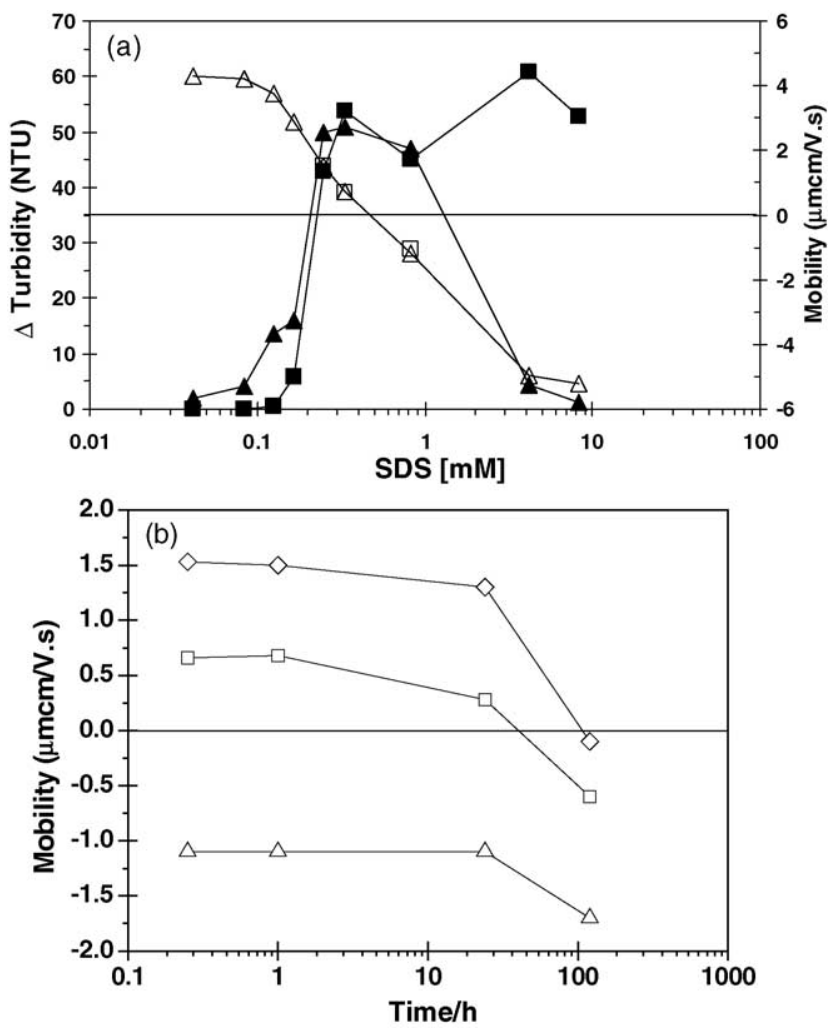

Fig. 2. (a) Electrophoretic mobility $(\triangle)$ and turbidity $(\mathbf{\Lambda})$ of PCMA-SDS aggregates formed through the STP-method in $10 \mathrm{mM} \mathrm{NaCl}$. For comparison we also display the turbidity curve for the PTS-series (ם) and the corresponding mobility data around the charge neutralization concentration $(\square)$. The PCMA concentration was $20 \mathrm{ppm}$. (b) The electrophoretic mobility of the PTS aggregates displayed in (a) is shown as a function of time: $(\diamond) 0.25 \mathrm{mM}$, ( $\square) 0.33 \mathrm{mM}$, and $(\triangle) 0.83 \mathrm{mM}$.

sequent aggregation. This is discussed in more detail in another publication [33].

\subsection{Dynamic light scattering measurements shortly after mixing}

The logarithms of the autocorrelation functions of two PCMA-SDS systems in $10 \mathrm{mM} \mathrm{NaCl}$ and formed through the STP-method, measured at different scattering angles, are shown in Fig. 3 (similar results were obtained for the PTS protocol). The results presented are obtained for samples in the regions where no precipitation was formed during the time span of our investigations $(1000 \mathrm{~h})$. The results indicate that the autocorrelation functions are close to single exponential functions and that the same diffusion coefficient is obtained at each scattering angle.

Fig. 4 displays the logarithm of the autocorrelation functions as a function of $\tau q^{2}$ (measured at $90^{\circ}$ ). It has to be pointed out that the figures also have included systems that show high turbidity (which consequently are influenced by multiple scattering effects) in order to show the effect of the increase in SDS concentration on the autocorrelation function. Even though the data obtained from these systems can- not be used for calculating, e.g. the hydrodynamic radius, the conclusion that large aggregates are formed is firm. One feature that is common for all plots is that the relaxation time increases dramatically when the SDS concentration is increased sufficiently to make the solution noticeably turbid. This happens in the vicinity of the charge neutralization concentration of the systems. However, while the aggregate size in samples prepared by the STP-method decreases significantly at SDS concentrations above the charge neutralization concentration, it continues to remain high for samples prepared through the PTS protocol (which is consistent with the results obtained using turbidity measurements). We also note the "tailing effect" at large $\tau q^{2}$-values (observed for some curves), which is a sign of the presence of some larger aggregates. Another interesting feature is the short relaxation times found at high SDS concentrations for samples prepared through the STPmethod. This indicates that the aggregates formed are very compact despite their high excess negative charge.

The hydrodynamic radii $\left(R_{\mathrm{h}}\right)$ evaluated from the dynamic light scattering data for PCMA-SDS aggregates in $10 \mathrm{mM}$ $\mathrm{NaCl}$, obtained through the different mixing methods (and measured $15 \mathrm{~min}$ after blending) are displayed in Fig. 5. Note that data that are corrupted by multiple scattering effects are omitted, but the large hydrodynamic radii close to the charge neutralization point are indicated by lines going towards high $R_{\mathrm{h}}$-values. We notice that at low SDS concentrations the sizes of the scattering units are considerably smaller when the PTS-method is used as compared with the STP-method. Thus, this is the reason behind the higher turbidity obtained with the STP-method in this low SDS concentration interval. These results also support the idea that some aggregates containing more than one polyelectrolyte chain are formed with the STP-method during the initial stage of mixing. At high SDS concentrations considerably larger aggregates are formed by the PTS-method as compared to the STP-method, consistent with the higher turbidity values found at these SDS concentrations with the PTS-method (data for the hydrodynamic radii are not shown). In this context it is useful to compare the size of the PCMA-SDS aggregates with that of the collapsed PCMA chain in concentrated salt solution. The $R_{\mathrm{h}}$-value for $20 \mathrm{ppm}$ PCMA in $1 \mathrm{M}$ $\mathrm{NaCl}$ was found to be $38 \mathrm{~nm}$ (marked with a dashed line in Fig. 5). For samples prepared by the PTS-method at low surfactant concentrations and for STP at high surfactant concentrations, the $R_{\mathrm{h}}$-value lies reasonably close to this value. Similar results have previously been reported by Guillot et al. [26] for the carboxymethylcellulose-DTAB and by us for the PVAm-SDS system [33] - but the present results differ from the previous ones in one respect. In their work Guillot et al. assigned the $R_{\mathrm{h}}$ of the investigated polyelectrolyte in $1 \mathrm{M}$ $\mathrm{NaCl}$, as the smallest size that the collapsed polyelectrolyte can obtain. However, we observe that further reduction in the polyelectrolyte size is possible by the addition of surfactant (this effect is most pronounced at $0.08 \mathrm{mM}$ ). The difference between carboxymethylcellulose-DTAB/DNA-DTAB and PCMA-SDS in this respect is most likely due to the 

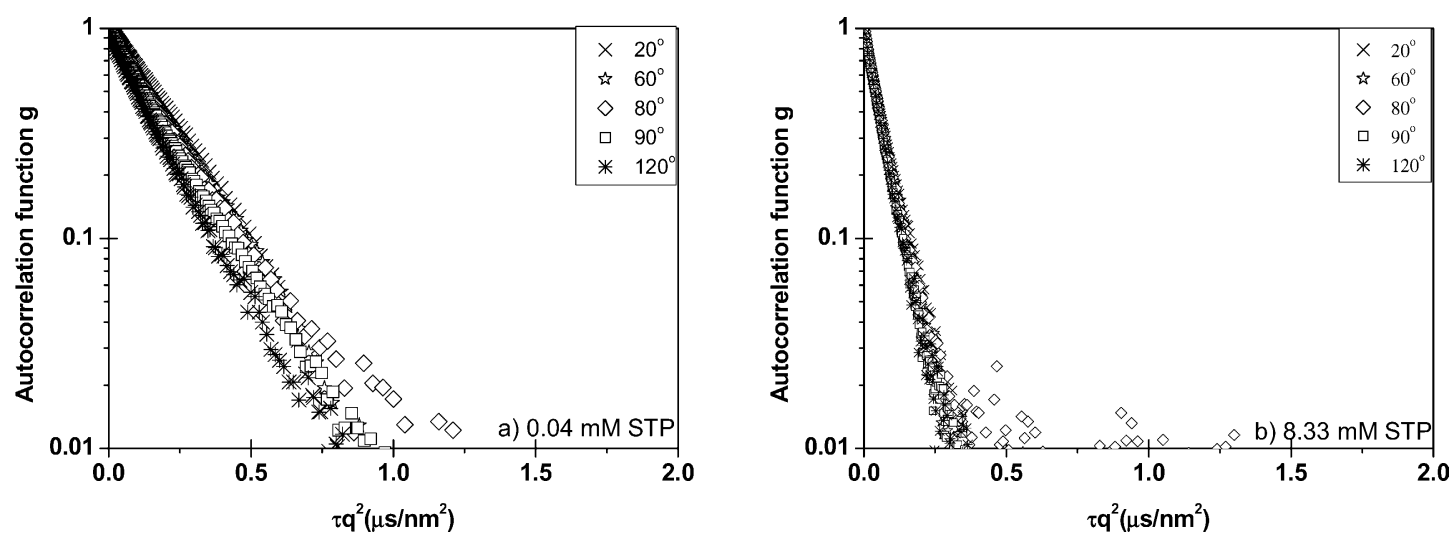

Fig. 3. The logarithm of the autocorrelation functions for PCMA-SDS aggregates in $10 \mathrm{mM} \mathrm{NaCl}$ obtained at different scattering angles vs. $\tau q^{2}$ : (a) $0.04 \mathrm{mM}$ STP and (b) $8.33 \mathrm{mM}$ STP. The PCMA concentration was $20 \mathrm{ppm}$.
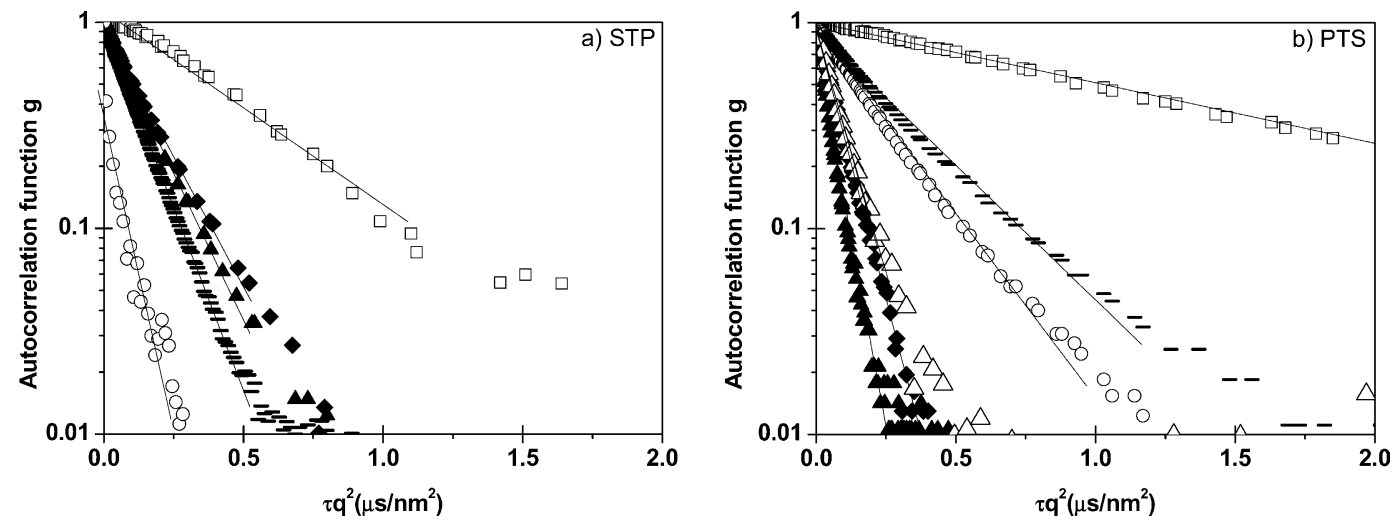

Fig. 4. The logarithm of the autocorrelation functions for some PCMA-SDS compositions (in $10 \mathrm{mM} \mathrm{NaCl}$, investigated 15 min after blending) measured at $90^{\circ}$ and plotted as a function of $\tau q^{2}$. (a) Samples prepared through the STP-method, (b) samples prepared through the PTS-method. The SDS concentrations

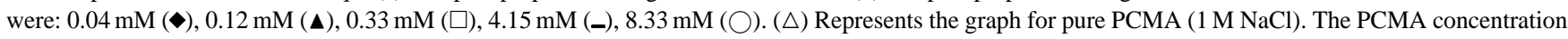
was $20 \mathrm{ppm}$.

significantly stiffer structures of carboxymethylcellulose and DNA compared to PCMA. We suggest that the much smaller size of the PCMA-SDS complex at low surfactant concentrations is due to the extra induction of hydrophobicity by SDS to the complex, which together with a reduction in the charge density, enable denser packing in the interior of the polyelectrolyte-surfactant complex. In fact, SANS and SAXS studies [29,31] have shown that the interior of the aggregates formed close to the charge neutralization point consists of cylindrical SDS micelles wrapped by polyelectrolyte arranged in a hexagonal organization. The water layer thickness, including the surfactant head group and the polyelectrolyte, between these structural units has been estimated to be $1.6 \mathrm{~nm}[31]$.

\subsection{Time evolution of the turbidity}

The stability of the dispersed polyelectrolyte-surfactant aggregates can be followed by measuring the turbidity as a function of time. We display our findings in Figs. 6-9, which show results obtained with the STP- and PTS-methods in solutions with different ionic strengths. Let us first discuss the data shown in Fig. 6a, obtained with the STP-method for the case with no added salt. It is clear that relatively low and stable turbidity values are obtained at concentrations up to $0.12 \mathrm{mM}$ SDS. In the SDS concentration interval $0.17-0.25 \mathrm{mM}$ the turbidity first increases (indicating forma-

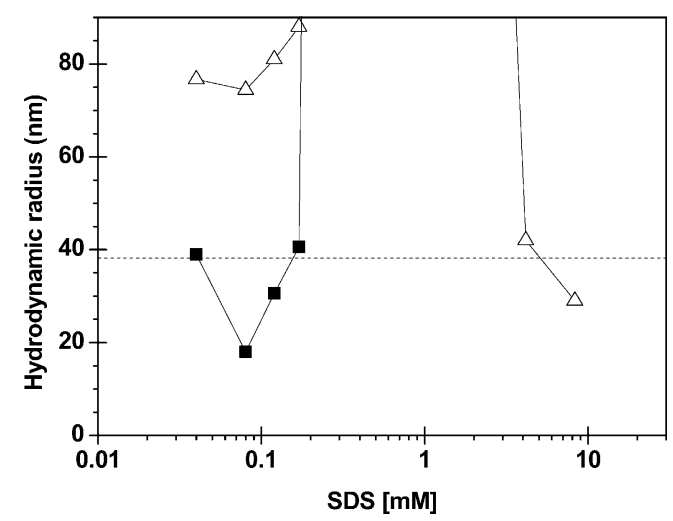

Fig. 5. The hydrodynamic radius of PCMA-SDS aggregates formed in $10 \mathrm{mM} \mathrm{NaCl}$, and obtained $15 \mathrm{~min}$ after the final blending. The samples were prepared by the STP-method $(\triangle)$ and the PTS-method ( $\square)$. The PCMA concentration was $20 \mathrm{ppm}$. The dashed line corresponds to the hydrodynamic radius of PCMA $(20 \mathrm{ppm})$ in $1 \mathrm{M} \mathrm{NaCl}$. 

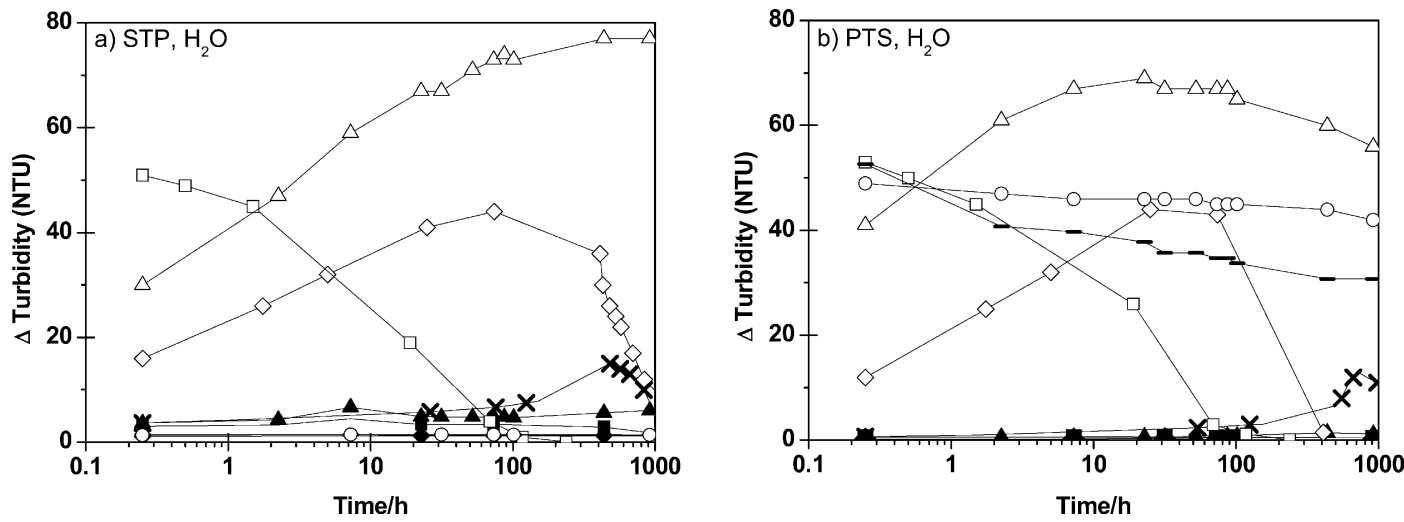

Fig. 6. Turbidity of solutions containing PCMA-SDS aggregates formed in pure water as a function of time. The samples were prepared by the STP-method (a), and the PTS-method (b). The PCMA concentration was $20 \mathrm{ppm}$. The SDS concentrations were: $0.04 \mathrm{mM}(\checkmark), 0.08 \mathrm{mM}(\mathbf{\square}), 0.12 \mathrm{mM}(\mathbf{\Lambda}), 0.17 \mathrm{mM}(\mathbf{X})$, $0.25 \mathrm{mM}(\diamond), 0.33 \mathrm{mM}(\square), 0.83 \mathrm{mM}(\triangle), 4.15 \mathrm{mM}(\mathbf{-}), 8.33 \mathrm{mM}(\bigcirc)$.
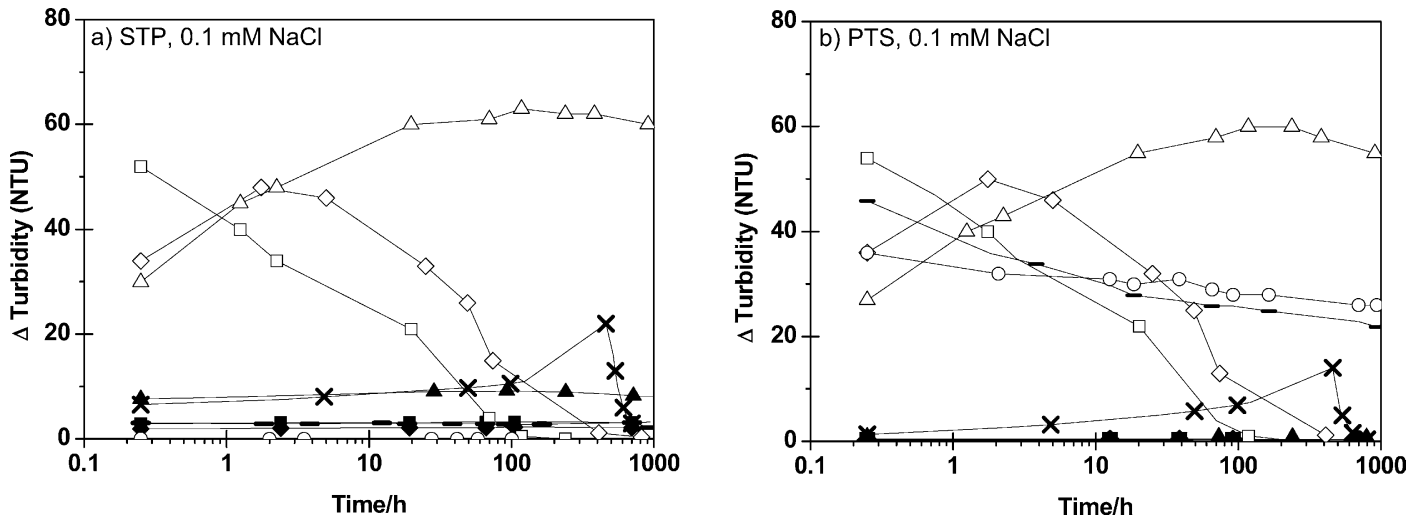

Fig. 7. Turbidity of solutions containing PCMA-SDS aggregates formed in $0.1 \mathrm{mM} \mathrm{NaCl}$ as a function of time. The samples were prepared by the STP-method (a), and the PTS-method (b). The PCMA concentration was $20 \mathrm{ppm}$. The SDS concentrations were: $0.04 \mathrm{mM}(\boldsymbol{\vee}), 0.08 \mathrm{mM}(\mathbf{\square}), 0.12 \mathrm{mM}(\mathbf{\Delta}), 0.17 \mathrm{mM}(\mathbf{X})$, $0.25 \mathrm{mM}(\diamond), 0.33 \mathrm{mM}(\square), 0.83 \mathrm{mM}(\triangle), 4.15 \mathrm{mM}(\mathbf{-}), 8.33 \mathrm{mM}(\bigcirc)$.
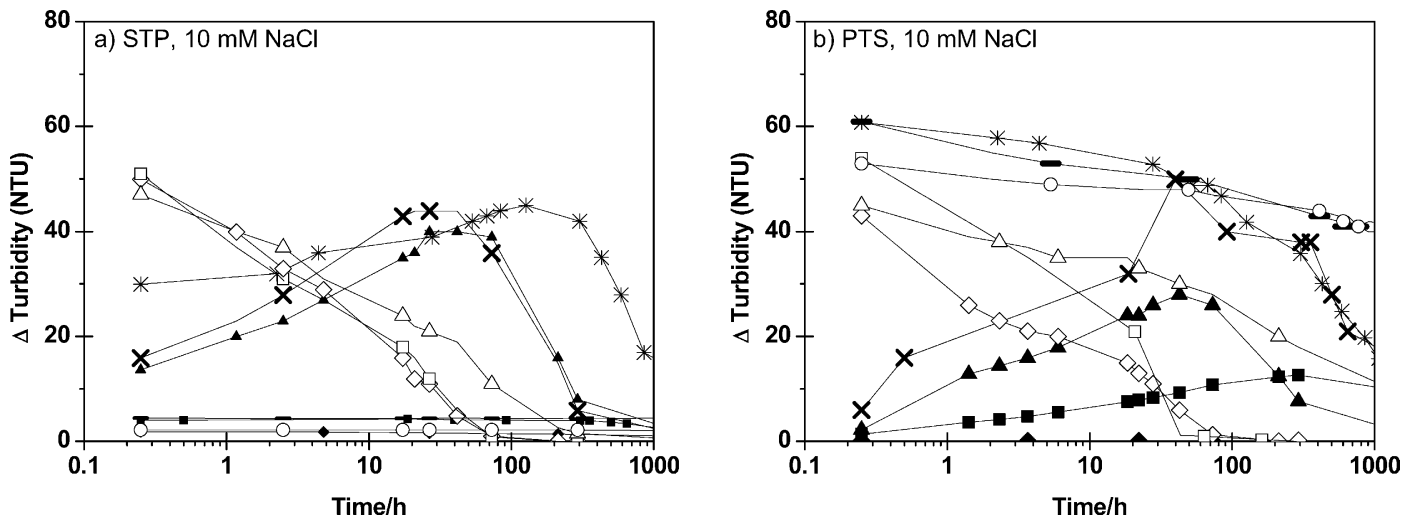

Fig. 8. Turbidity of solutions containing PCMA-SDS aggregates formed in $10 \mathrm{mM} \mathrm{NaCl}$ as a function of time. The samples were prepared by the STP-method (a), and the PTS-method (b). The PCMA concentration was $20 \mathrm{ppm}$. The SDS concentrations were: $0.04 \mathrm{mM}(\boldsymbol{\downarrow}), 0.08 \mathrm{mM}(\boldsymbol{\square}), 0.12 \mathrm{mM}(\mathbf{\Delta}), 0.17 \mathrm{mM}(\mathbf{X})$, $0.25 \mathrm{mM}(\diamond), 0.33 \mathrm{mM}(\square), 0.83 \mathrm{mM}(\triangle), 1.66 \mathrm{mM}(*), 4.15 \mathrm{mM}\left(\_\right), 8.33 \mathrm{mM}(\bigcirc)$. 

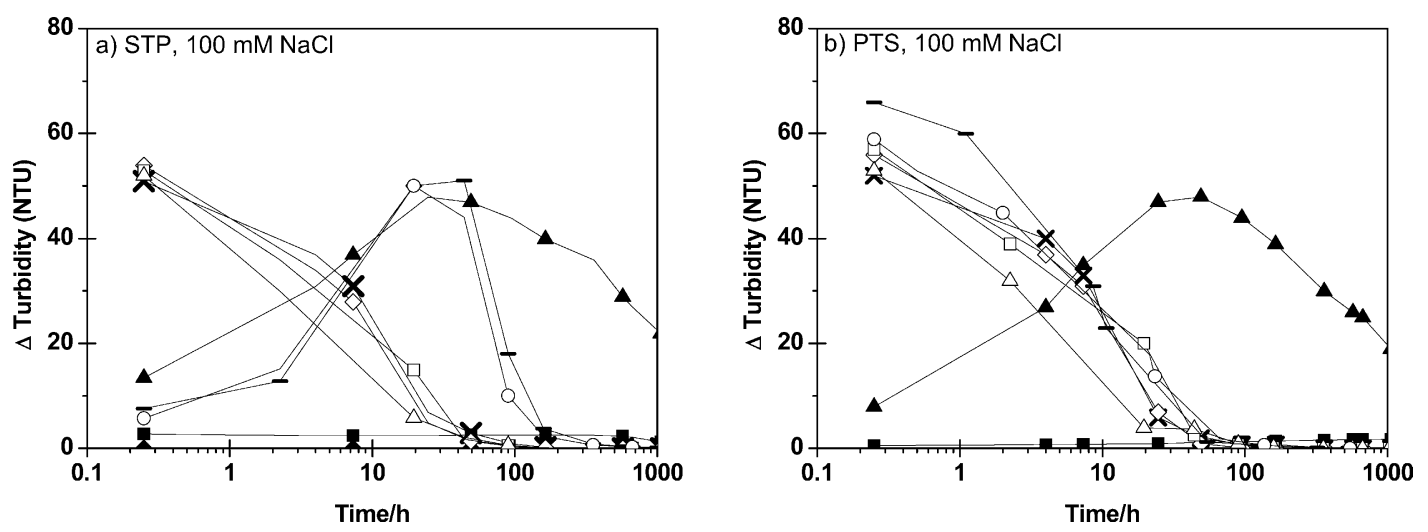

Fig. 9. Turbidity of solutions containing PCMA-SDS aggregates formed in $100 \mathrm{mM} \mathrm{NaCl}$ as a function of time. The samples were prepared by the STP-method (a), and the PTS-method (b). The PCMA concentration was $20 \mathrm{ppm}$. The SDS concentrations were: $0.04 \mathrm{mM}(\boldsymbol{\vee}), 0.08 \mathrm{mM}(\mathbf{\square}), 0.12 \mathrm{mM}(\mathbf{\Lambda}), 0.17 \mathrm{mM}(\times)$, $0.25 \mathrm{mM}(\diamond), 0.33 \mathrm{mM}(\square), 0.83 \mathrm{mM}(\triangle), 4.15 \mathrm{mM}(-), 8.33 \mathrm{mM}(\bigcirc)$.

tion of larger aggregates) and then decreases again with time. At an SDS concentration of $0.33 \mathrm{mM}$ the turbidity decreases rapidly with time. Visual inspection shows that sediment is formed and thus a macroscopic phase separation occurs rapidly close to the charge neutralization point. A further increase in SDS concentration to $0.8 \mathrm{mM}$ again results in a solution where the turbidity initially is increasing with time. For even longer times than illustrated in Fig. 6a, the turbidity starts to decrease again. For instance after $1000 \mathrm{~h}$ the turbidity for the solution containing $0.8 \mathrm{mM}$ was 77 , which decreased to 70 after $6000 \mathrm{~h}$. Hence, two processes occur; aggregation of polyelectrolyte-surfactant complexes followed by sedimentation when the aggregates have grown to a sufficient size. Low and stable turbidity values are obtained at even higher SDS concentrations, indicating formation of stable dispersions of polyelectrolyte-surfactant aggregates. The general features of the time evolution of the turbidity are mostly the same when using the PTS- and STP-method. The most noticeable difference is found at high surfactant concentrations where the high turbidity obtained with the PTS-method decreases slowly with time.

The binding isotherm for SDS on PCMA is not available but the free SDS concentration at the charge neutralization point can be estimated from the SDS concentration $(0.33 \mathrm{mM})$ and the polyelectrolyte segment concentration $(0.13 \mathrm{mM})$ to be about $0.20 \mathrm{mM}$. Thus, adding $\mathrm{NaCl}$ to a concentration of $0.1 \mathrm{mM}$ does not change the ionic strength drastically, and it is thus not expected to influence the stability of the aggregates significantly above the charge neutralization point. Comparing the data in Fig. 6 (no added salt) with the data in Fig. $7(0.1 \mathrm{mM} \mathrm{NaCl})$ confirms this. However, we note that the sedimentation rate close to the charge neutralization point, as estimated from the decrease in turbidity with time, is increased in presence of even such a small $\mathrm{NaCl}$ concentration as $0.1 \mathrm{mM}$.

Adding $\mathrm{NaCl}$ to a concentration of $10 \mathrm{mM}$ does influence the ionic strength of the solutions significantly, and this also affects the time evolution of the turbidity. As seen in Fig. 8, the range of the colloidally unstable region has clearly in- creased as compared to at lower $\mathrm{NaCl}$ concentrations. However, stable values for the turbidity are still obtained at the lowest (both STP- and PTS-method) and highest surfactant concentrations (only the STP-method). A further increase in the $\mathrm{NaCl}$ concentration to $100 \mathrm{mM}$, Fig. 9, induces instability also at the highest surfactant concentration independent of the preparation method, whereas the stability remains at low surfactant concentrations.

The instability region as a function of SDS and $\mathrm{NaCl}$ concentration is displayed in Fig. 10. The diagrams show regions where sediment has formed within $1000 \mathrm{~h}$ after the mixing of PCMA and SDS. The observation that the colloidally unstable region increases with ionic strength is a clear evidence for repulsive electrostatic double-layer forces being of importance for the stability of the aggregates formed, which are kinetically stabilized by the presence of excess charges. We note that for the STP-method the electrolyte concentration has a smaller influence on the border between stability and instability at low surfactant concentrations, where the aggregates have a net positive charge, compared to at high surfactant concentrations where the surfactants are present in excess in the aggregates. These results indicate that there is an efficiency difference between excess polyelectrolyte and surfactant in stabilization of the aggregates. Let us rationalize this by considering the internal structure of the aggregates. This has previously been determined with SANS [29] and SAXS [31] for the PCMA-SDS system prepared by the PTS-method without added salt. It was shown that the internal structure of the aggregates is characterized by a 2D-hexagonal organization due to packing of cylindrical SDS micelles wrapped by the polyelectrolyte. This internal organization of the aggregates persists both in the sediment and in excess of SDS (at least up to a three-fold excess) and in excess of polyelectrolyte (at least up to a three-fold excess) in the colloidally stable regions. The large magnitude and the negative sign of the mobility of the colloidally stable aggregates in excess SDS, combined with the stability of the internal structure to excess SDS, give us strong reasons to suggest that the excess SDS is accumulated as a skin on the outside of the aggregates. 

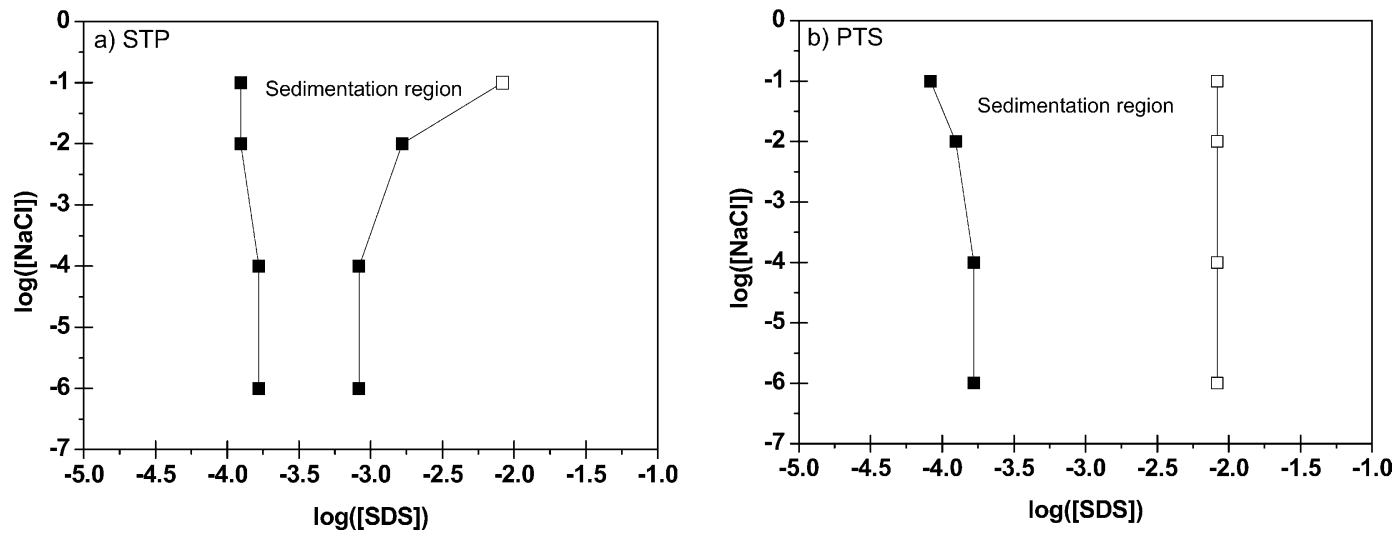

Fig. 10. The colloidally unstable/sedimentation region ( $1000 \mathrm{~h}$ after mixing) for solutions containing PCMA-SDS aggregates prepared through the STP-method (a) and the PTS-method (b). The $\mathrm{NaCl}$ and the SDS concentrations are both expressed in molar. The unfilled symbols represent the highest SDS concentration investigated. Here a precipitate was formed. Whether a precipitate is formed also at higher SDS concentration is likely but not determined. The points shown at $10^{-6} \mathrm{M} \mathrm{NaCl}$ were in reality determined in pure water (no added salt).

It seems plausible that during a collision event between two aggregates with excess SDS it is comparatively easy for the surfactant molecules accumulated at the surface of the aggregates to desorb, which would facilitate aggregation. The increased ionic strength reduces the energy barrier and makes these events more likely. On the other hand, at low surfactant concentrations the increased ionic strength also reduces the energy barrier during a collision event, but in this case there is no simple way for the aggregates to get rid of their excess charges, which are chemically bound to the polyelectrolyte, during a collision event. This can occur by additional surfactant adsorption to the aggregates during collision, but this is a slow event in dilute surfactant solutions. A complication may be that the internal structure of the PCMA-SDS aggregates is affected by the $\mathrm{NaCl}$ concentration. However, no SANS or SAXS data are available at higher $\mathrm{NaCl}$ concentrations, and consequently we cannot discuss this further.

\subsection{Time evolution of the electrophoretic mobility}

In Fig. $2 b$ the time evolution of the electrophoretic mobility of some PCMA-SDS aggregates (formed through PTS in
$10 \mathrm{mM} \mathrm{NaCl}$ ) around the charge neutralization concentration (CNC) of the system is shown. Clearly, the mobility of the aggregates decreases slightly with time, which partly can be rationalized by the continuous aggregation of the PCMA-SDS aggregates. However, we note that the mobility of the aggregates actually may change sign, from positive to negative, with time. Hence, it appears that an additional slow incorporation of SDS in the aggregates occurs, a process that is not well understood at the time of writing.

\subsection{Time evaluation of PCMA-SDS solutions by dynamic light scattering}

Fig. 11 illustrates how the $R_{\mathrm{h}}$-values of PCMA-SDS aggregates, in $10 \mathrm{mM} \mathrm{NaCl}$, change with time. Only data for samples with small aggregates, avoiding complications due to multiple scattering, are shown. Aggregates with a size that does not change with time are formed at low surfactant concentrations, up to about $0.08 \mathrm{mM}$ SDS, using the STP-method (Fig. 11a). This is consistent with the turbidity measurements and the notion of formation of colloidally stable aggregates. Apparently once formed these aggregates,
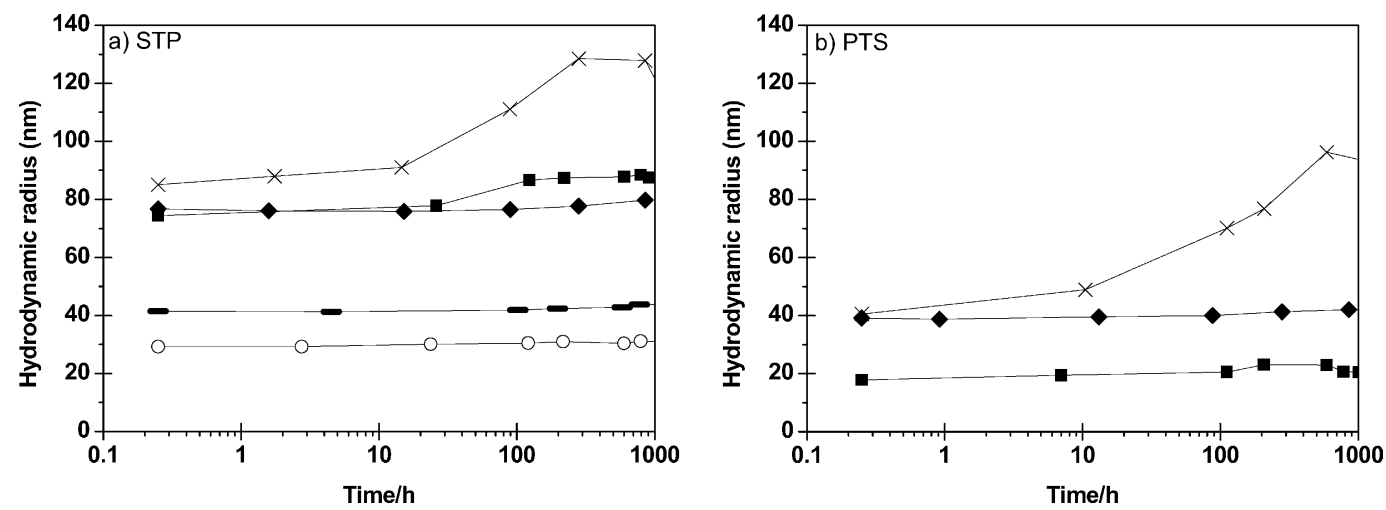

Fig. 11. The hydrodynamic radius vs. time of PCMA-SDS aggregates formed by the STP-method (a) and the PTS-method (b) in 10 mM NaCl. The PCMA concentration was $20 \mathrm{ppm}$. The SDS concentrations were: $0.04 \mathrm{mM}(\bullet), 0.08 \mathrm{mM}(\boldsymbol{\square}), 0.17 \mathrm{mM}(\mathbf{X}), 4.15 \mathrm{mM}(\mathbf{-}), 8.33 \mathrm{mM}(\bigcirc)$. 
Table 1

Polydispersity of PCMA-SDS aggregates in $10 \mathrm{mM} \mathrm{NaCl}$ measured at $0.25 \mathrm{~h}$ and ca. $1000 \mathrm{~h}$ after mixing

\begin{tabular}{lll}
\hline SDS $(\mathrm{mM})$ & Polydispersity $(0.25 \mathrm{~h})$ & Polydispersity $(\mathrm{ca} .1000 \mathrm{~h})$ \\
\hline 0.04 STP & 0.22 & 0.19 \\
0.04 PTS & 0.10 & 0.10 \\
0.08 STP & 0.25 & 0.26 \\
0.08 PTS & 0.15 & 0.17 \\
4.15 STP & 0.14 & 0.15 \\
8.33 STP & 0.17 & 0.18 \\
\hline
\end{tabular}

which are considerably larger than those formed by the PTSmethod remain stable during a period of at least $1000 \mathrm{~h}$. Hence, since the difference in aggregate properties for the two methods remains for prolonged times it is clear that we observe non-equilibrium trapped states (the true equilibrium state remains unknown). However, when the SDS concentration is increased to $0.17 \mathrm{mM}$ the size of the aggregates increases with time. Hence, flocculation clearly occurs, and as a consequence the turbidity increases. At high surfactant concentrations, at and above $4 \mathrm{mM} \mathrm{SDS}$, the size of the aggregates is again nearly invariant with time. For samples prepared by the PTS-method (Fig. 11b) we notice the same general trends as for the STP samples.

From an industrial point of view it may be of importance to produce aggregates not only with controlled size and good colloidal stability, but also low polydispersity. In Table 1 we present the polydispersity of some systems (in $10 \mathrm{mM}$ $\mathrm{NaCl}$ ) prepared through the STP and PTS-methods, determined at two different "equilibration" times $(0.25 \mathrm{~h}$ and ca. $1000 \mathrm{~h}$ after the final mixing of the ingredients). Two trends in the results can be distinguished. The first observation is that both methods give low polydispersity, which remains more or less constant with time. Our second observation is that the STP-method seems to give higher polydispersity than the PTS-method in the low SDS concentration region, which we suggest is due to the non-equilibrium effects previously discussed.

\section{Conclusions}

In this paper we have addressed order of addition effects on the association between a highly charged cationic polyelectrolyte and an oppositely charged surfactant. The mixing methods investigated were "STP" through which the surfactant was added to the polyelectrolyte solution, and "PTS" which involved the addition of the polyelectrolyte to the surfactant solution. It was shown that the mixing procedure has a significant effect on the bulk association process, in particular at high surfactant concentrations but also at low surfactant concentrations close to the border between colloidal stability and instability. The order of addition effect can be traced to the initial non-homogeneous distribution of the component added last. As a result larger aggregates are formed with the STP-method at low surfactant concentrations and with the PTS-method at high surfactant concentrations. The difference between the solutions obtained with the two mixing methods persists for prolonged times, which demonstrates the existence of trapped non-equilibrium states. A consequence of this is that it should be possible to tune the size of the aggregates formed by carefully controlling the mixing process. It was also shown that the surfactant range over which colloidally unstable aggregates are formed increases with increasing salt concentration, an observation that is consistent with an electrostatic stabilization mechanism. In particular, the instability region for the small aggregates formed by the STP-method increased towards the high surfactant side, an observation that was rationalized by the charge regulation possibilities of the aggregates. In excess surfactant the aggregates have an outer skin of surfactants that can be displaced during a collision event, facilitating their aggregation. No equally effective charge regulation process exists for positively charged aggregates where the polyelectrolyte provides the excess charges. Dynamic light scattering measurements demonstrated that colloidally stable systems indeed were formed in excess surfactant and excess polyelectrolyte. In some cases the aggregates formed had a remarkably small polydispersity. Closer to the charge neutralization point the size of the aggregates was found to increase with time and ultimately a macroscopic phase separation occurred.

\section{Acknowledgment}

This work was supported by the Swedish Research Council (VR).

\section{References}

[1] A. Svensson, L. Piculell, B. Cabane, P. Ilekti, J. Phys. Chem. B 106 (2002) 1013-1018.

[2] A. Malovikova, K. Hayakawa, J.C.T. Kwak, J. Phys. Chem. 88 (1984) 930-1933.

[3] I. Satake, T. Takahashi, K. Hayakawa, T. Maeda, M. Aoyagi, Bull. Chem. Soc. Jpn. 63 (1990) 926-928.

[4] T. Wallin, P. Linse, J. Phys. Chem. 100 (1996) 17873-17880.

[5] Y. Li, P.L. Dubin, in: C.A. Herb, R.K. Prud'homme (Eds.), Structure and Flow in Surfactant Solutions, vol. 578, American Chemical Society, Washington, DC, 1994, pp. 320-336.

[6] H. Okuzaki, Y. Osada, Macromolecules 27 (1994) 502-506.

[7] T. Wallin, P. Linse, J. Phys. Chem. 101 (1997) 5506-5513.

[8] I. Iliopoulos, Curr. Opin. Colloid Interf. Sci. 3 (1998) 493-498.

[9] T. Wallin, P. Linse, Langmuir 12 (1996) 305-314.

[10] R. Mészaros, L. Thompson, M. Bos, I. Varga, T. Gilanyi, Langmuir 19 (2003) 609

[11] M.A. Winnik, S.M. Bystryak, C. Chassenieux, Langmuir 16 (2000) 4495-4510.

[12] P.M. Claesson, U.R.M. Kjellin, in: B. Binks (Ed.), Modern Characterization Methods of Surfactant Systems, Surfactant Science Series, vol. 83, pp. 255-333, Marcel M. Langmuir 16 (2000) 1951.

[13] S.M.A. Cohen, G.J. Fleer, Ann. Rev. Mater. Sci. 26 (1996) 463-500.

[14] M.A.G. Dahlgren, Å. Waltermo, E. Blomberg, P.M. Claesson, L. Sjöström, T. Åkesson, B. Jönsson, J. Phys. Chem. 97 (1993) 11769-11775. 
[15] M.A.G. Dahlgren, H.C.M. Hollenberg, P.M. Claesson, Langmuir 11 (1995) 4480-4485.

[16] S.A. Sukhishvili, A. Dhinojwala, S. Granick, Langmuir 15 (1999) 8474-8482.

[17] J. Chen, J.A. Heitmann, M.A. Hubbe, Colloids Surf. A: Physicochem. Eng. Aspects 223 (2003) 215-230.

[18] A. Dedinaite, P.M. Claesson, M. Bergström, Langmuir 16 (2000) 5257-5266.

[19] E.S. Pagac, D.C. Prieve, R.D. Tilton, Langmuir 14 (1998) 2333-2342.

[20] A.D. Braem, D.C. Prieve, R.D. Tilton, Langmuir 17 (2001) 883-890

[21] K.D. Berglund, T.M. Przybycien, R.D. Tilton, Langmuir 19 (2003) 2705.

[22] K.D. Berglund, T.M. Przybycien, R.D. Tilton, Langmuir 19 (2003) 2714.

[23] S.B. Velegol, R.D. Tilton, Langmuir 17 (2001) 219.

[24] S.B. Velegol, R.D. Tilton, J. Colloid Interf. Sci. 249 (2002) 282-289.

[25] O.J. Rojas, P.M. Claesson, K.D. Berglund, R.D. Tilton, Langmuir 20 (2004) 3221.
[26] S. Guillot, M. Delsanti, S. Désert, D. Langevin, Langmuir 19 (2003) 230-237.

[27] S. Guillot, D. McLoughlin, N. Jain, M. Delsanti, D. Langevin, J. Phys.: Condens. Matter 15 (2003) S219-S224.

[28] M. Antonietti, J. Conrad, A. Thünemann, Macromolecules 27 (1994) 6007-6011.

[29] P.M. Claesson, M. Bergström, A. Dedinaite, M. Kjellin, J.F. Legrand, I. Grillo, J. Phys. Chem. B 104 (2000) 1168911694.

[30] S. Zhou, H. Hu, C. Burger, B. Chu, Macromolecules 34 (2001) 1772 .

[31] M. Bergström, U.R.M. Kjellin, P.M. Claesson, J.S. Pedersen, M.M. Nielsen, J. Phys. Chem. B 106 (2002) 11412-11419.

[32] M. Bergström, U.R.M. Kjellin, P.M. Claesson, I. Grillo, J. Phys. Chem. B 108 (6) (2004) 1874-1881.

[33] A. Naderi, P.M. Claesson, submitted for publication.

[34] W. Brown, Light Scattering Principles and Development, Clarendon Press, Oxford, 1996.

[35] A. Dedinaite, P.M. Claesson, Langmuir 16 (2000) 1951-1959. 